\title{
Dynamic structure of electrons in Be metal by inelastic x-ray scattering spectroscopy
}

\author{
W. Schülke, H. Nagasawa, S. Mourikis, and A. Kaprolat \\ Institute of Physics, University of Dortmund, D-4600 Dortmund 50, Federal Republic of Germany
}

(Received 8 May 1989)

\begin{abstract}
The dynamic structure factor $S(\mathbf{q}, \omega)$ of electrons in Be metal was measured with 1-eV resolution by means of inelastic scattering of synchrotron x radiation both on single crystals for a large number of different directions of $\mathbf{q}$ (among others, $\mathbf{q} \| \mathbf{g}_{100}, \mathbf{g}_{110}, \mathbf{g}_{101}, \mathbf{g}_{001}$ ) and on polycrystalline samples with 0.45 a.u. $<q<1.69$ a.u. The experimental results were compared with model calculations, which go beyond the random-phase approximation. The overall shape of experimental $S(\mathbf{q}, \omega)$ can be understood when taking into account appropriately both state-dependent quasiparticle lifetime and local-field corrections. The strongly q-orientation-dependent fine structure of $S(\mathbf{q}, \omega)$ can be interpreted as being due to excitation gaps, which are generated by transitions to final states on Bragg planes. This type of fine structure can be utilized to get information about the band structure in a very direct manner. There is every indication that also the less q-orientation-dependent fine structure found in experimental $S(\mathbf{q}, \omega)$ is induced by ion-electron interaction, although another origin (electron-electron interaction) cannot be ruled out completely.
\end{abstract}

\section{INTRODUCTION}

Inelastic $\mathrm{x}$-ray scattering spectroscopy (IXSS) is best suited to obtain information about the dynamic structure factor $S(\mathrm{q}, \omega)$ of electrons in solids. ${ }^{1}$ The range of momentum (q) and energy $(\omega)$ transfer is wide enough so that short-range correlation can be investigated. Already early IXSS measurements of $S(\mathrm{q}, \omega)$ on simple metals have indicated the break down of the random phase approximation (RPA) for $q>q_{c}\left(q_{c}=\right.$ plasmon cutoff vector). ${ }^{2}$ Above all, a double-peak structure in $S(\mathbf{q}, \omega)$ found in simple metals ( $\mathrm{Li}, \mathrm{Be}, \mathrm{Al}$ ) (Refs. 3 and 4) and graphite has induced a controversy about its origin. Because the double-peak structure has been found for very different systems roughly at the same energy position when scaling the energy transfer to the Fermi energy, ${ }^{2}$ many authors tend to attribute this fine structure to a universal property of the strongly correlated electron liquid. But not all of them succeeded in reproducing the double-peak structure in their calculations of $S(\mathbf{q}, \omega)$ for an homogeneous electron liquid. The first step to go beyond RPA was done by Vashishta and Singwi ${ }^{6}$ by introducing local-field correction. But the static approximation of the local-field correction could not result in a double-peak structure of $S(q, \omega)$. Later on, neither Pajanne's ${ }^{7}$ formalism, based on a boson formulation of interacting fermions, nor the rigorous dielectric treatment of Utsumi and Ichimaru, ${ }^{8}$ which satisfied, besides others, the equation of continuity and the frequency-moment sum rules, could reproduce the double peak. The application of Mori's memoryfunction approach by De Raedt and De Raedt ${ }^{9}$ succeeded in getting a double-peak structure. But they had to represent the static structure factor $S(q)$ by means of parameters adjusted in a way to qualitative agreement with experiment. Green et al. ${ }^{10}$ have treated the electron liquid by taking into account rigorously the short-range Coulomb interaction of electron pairs and by satisfying all conservation sum rules. They have obtained some fine structure of $S(\mathrm{q}, \omega)$ but in disagreement with experiment $^{11}$ and without giving a sufficient physical explanation of how this fine structure originates. Only by introducing a state-dependent lifetime via the imaginary part of the self-energy, as first done by Mukhopadhyay et al., ${ }^{12}$ could a double-peak fine structure of $S(\mathbf{q}, \omega)$ be reproduced. Objections against this approach, which is equivalent to a relaxation-time approximation, have been put forward among others by Mukhopadhyay and Sjölander ${ }^{13}$ pointing to the fact that the continuity equation seems to be violated. Subsequently Awa et al. ${ }^{14}$ Niklasson et al. ${ }^{15}$ and Rahman and Vignale ${ }^{16}$ have stressed the following physical grounds for the doublepeak structure, which appears by introducing higherorder excitations such as two-pair excitations and onepair-one-plasmon excitations within the framework of the quasi-one-pair excitation approximation: ${ }^{14}$ It is the steep rise of the reciprocal lifetime, which occurs when the quasiparticle momenta become large enough, so that the quasiparticles start decaying by emitting a plasmon. In this way the $S(\mathbf{q}, \omega)$ spectra are broadened beyond a critical value of $\omega$, so that the peak is shifted to lower $\omega$ compared with the RPA-peak position. This general gross feature of the simple metal $S(\mathbf{q}, \omega)$ has been well established by IXSS experiments, ${ }^{11,17-20}$ which have followed the findings of Platzman and Eisenberger. ${ }^{4}$ To what extent the two $\omega$ domains with very different lifetime broadening are well separated by a dip, so that a real double-peak structure occurs rather than a onepeak-one-shoulder structure, seems to depend strongly on the model used for the lifetime in the theoretical studies (Refs. 12, 14-16, and 20. The more realistic these models are, the more the dip is flattened or completely washed out in the theoretical calculations. Moreover, Niklasson ${ }^{21}$ has pointed out that the lifetime-induced double-peak structure disappears nearly completely when 
the frequency dependence of the corresponding real part of the self-energy is taken into account. $\mathrm{Ng}$ and $\mathrm{Da}$ browski ${ }^{22}$ came to the same conclusion. (The calculations of Ref. 14 strongly deviate from those of Refs. 12, 15, 16, and 20 , with respect to the dip position, the dip dispersion, and the dip depth. Since the theoretical results of Refs. 12, 15, 16, and 20 are in fairly good agreement one with another, the discrepancy with Ref. 14 must either be left unexplained or attributed to calculation errors in Ref. 14.) When comparing the calculated dip positions, if any, with the dip position of corresponding experimental results as obtained with polycrystalline samples, the situation becomes still more confusing. The Li result of Priftis et $a l .{ }^{18}$ is not far from the theoretical result of Rahman and Vignale ${ }^{16}$ (step model for the inverse lifetime), but is very far from the calculated dip position of Awa et al. ${ }^{14}$ The Be result of Vradis and Priftis ${ }^{19}$ fits Awa's ${ }^{14}$ calculations much better than those both of Rahman and Vignale $^{16}$ (a more realistic model for the inverse lifetime) and of Mukhopadhyay et al. ${ }^{12}$

Therefore, it seems advisable to look for another origin of the $S(\mathbf{q}, \omega)$ fine structure, namely the electron-ion interaction, where we keep in mind that the gross features of the simple metal $S(\mathbf{q}, \omega)$ (deviation of the overall shape from RPA) are well understood within the framework of the quasi-one-pair excitation approximation by representing short-range correlations by a local-field correction ${ }^{6}$ and higher-order excitations by a lifetime of the quasiparticle states. Considering of electron-ion interaction to be the origin of the remaining fine structure of $S(q, \omega)$ is mainly suggested by recent high-resolution measurements ${ }^{11,20}$ on $\mathrm{Li}$ and $\mathrm{Be}$ metal, which have revealed a much richer fine structure of $S(\mathbf{q}, \omega)$ than that seen in former low-resolution experiments. Also, the full bandstructure calculation of $S(\mathbf{q}, \omega)$ for $\mathrm{Be}$ of Taut and Hanke, ${ }^{23}$ to the knowledge of the authors the only one on simple metals, is rather encouraging, since, for intermediate momentum transfer $\left(q_{c}<q<2 k_{F}\right)$, a rich and strong fine structure has been found within the limits of the RPA, which has been attributed to electron-ion interaction. These findings were in disagreement with a very general statement of Pendey et al. ${ }^{24}$ that even in the most favorable cases band structure will modify the free electron $S(\mathbf{q}, \omega)$ by only a few percent. This statement has often been used as an argument in favor of the correlation-induced origin of the double-peak structure.

Therefore, it seemed worthwhile to investigate $S(\mathbf{q}, \omega)$ of beryllium metal experimentally with high-resolution, high-statistical accuracy and for a large range of momentum transfer $q$ in order to get enough experimental data which can be confronted with corresponding model calculations. Beryllium was chosen, since it is, on the one hand, a simple metal, so that $d$ electrons are not involved, but, on the other hand, it exhibits considerable deviations from free-electron behavior, so that stronger effects of electron-ion interaction could be expected than in former measurements on $\mathrm{Li}^{11}$ Part of the Be measurements have already been published. ${ }^{20}$

The rest of the paper is organized as follows: In Sec. II A a few fundamentals of IXSS are presented together with references, which can be consulted for more details.
In Sec. II B the effect of lattice-induced gaps in the oneelectron excitation spectrum on IXSS is shown at somewhat more length. Section II C gives a short survey of the experimental setup and of data processing, including references for more detailed presentation. In Sec. III the results of the $S(\mathbf{q}, \omega)$ measurements on Be are summarized. The discussion of the results in Sec. IV is arranged as follows: First (Sec. IV A), the measurements on polycrystalline $\mathrm{Be}$ are confronted with model calculations for an homogeneous system in order to stress the importance of both lifetime effects and local-field corrections for the understanding of the overall shape of $S(\mathbf{q}, \omega)$. Secondly (Sec. IV B), the fine structure in the single crystal results and its dependence on the direction of $\mathbf{q}$ is compared with model calculations of $S(\mathbf{q}, \omega)$ so that the existence of excitation gaps with final states on zone boundaries becomes evident. On the basis of this interpretation of essential parts of the $S(\mathbf{q}, \omega)$ fine structure, a method of probing the ground-state band structure by means of IXSS is proposed. The remaining less-q-orientationdependent fine structure, which cannot be simply interpreted within the limits of a two-band model, is discussed in Sec. IV C, where both realistic band-structure calculations of $S(\mathbf{q}, \omega)$ and model calculations going beyond the two-band model are brought into play. Finally, in Sec. V conclusions about the most probable origin of the $S(\mathrm{q}, \omega)$ fine structure are drawn from the comparison of experiment with calculations.

\section{INELASTIC X-RAY SCATTERING SPECTROSCOPY}

\section{A. Fundamentals}

In the following, inelastic-scattering processes are considered, where a photon of energy $\hbar \omega_{1}$, wave vector $\mathbf{K}_{1}$, and polarization $\widehat{e}_{1}$ is scattered by the electron system into a photon of energy $\hbar \omega_{2}$, wave vector $\mathbf{K}_{2}$, and polarization $\widehat{e}_{2}$, so that the energy

$$
\hbar \omega=\hbar\left(\omega_{1}-\omega_{2}\right)
$$

and the momentum

$$
\hbar \mathbf{q}=\hbar\left(\mathbf{K}_{1}-\mathbf{K}_{2}\right) ; \quad \hbar q \approx 2 \hbar K_{1} \sin (\theta / 2)
$$

are transferred to the scattering system, where $\theta$ is the scattering angle. The second part of Eq. (2) holds for $\omega<<\omega_{1}$.

Therefore, a typical IXSS experiment consists in producing a well-collimated beam of monochromatic primary photons, in selecting a certain solid angle element $d \Omega$ of the scattered beam, so that $\mathbf{q}$ is fixed, and in analyzing this part of the scattered radiation to a resolution $d \omega_{2}$. Strictly speaking, one is measuring the doubledifferential cross section (DDCS) $d^{2} \sigma / d \Omega d \omega_{2}$.

Within the limits of a first-order perturbation treatment, the DDCS is related to the dynamic structure factor $S(\mathbf{q}, \omega)$ of the scattering system: ${ }^{25}$

$$
d^{2} \sigma / d \Omega d \omega_{2}=\left(\hat{e}_{1} \cdot \widehat{e}_{2}\right)^{2} r_{0}^{2}\left(\omega_{2} / \omega_{1}\right) S(\mathbf{q}, \omega),
$$

where $r_{0}$ is the classical electron radius. 
As long as the intensity of the primary photon beam is not measured, the DDCS is determined on a relative scale. Utilizing the $f$ sum rule

$$
\int_{0}^{\infty} S(\mathbf{q}, \omega) \omega d \omega=\hbar q^{2} / 2 m,
$$

the measured DDCS can be brought to an absolute scale. The fluctuation-dissipation theorem ${ }^{26}$ connects $S(\mathrm{q}, \omega)$ with the dielectric response function $\operatorname{Im} \epsilon^{-1}(\mathbf{q}, \omega)$ via

$$
S(\mathbf{q}, \omega)=-\left(\hbar q^{2} / 4 \pi^{2} e^{2} n\right) \operatorname{Im} \epsilon^{-1}(\mathbf{q}, \omega),
$$

where $n$ is the electron density.

Since $S(\mathbf{q}, \omega)$ and $\operatorname{Im} \epsilon^{-1}(\mathbf{q}, \omega)$ can be measured on an absolute scale, the real and the imaginary parts of the dielectric function

$$
\epsilon(\mathbf{q}, \omega)=\epsilon_{1}(\mathbf{q}, \omega)+i \epsilon_{2}(\mathbf{q}, \omega)
$$

can also be deduced from experiment by means of Kramers-Kronig analysis, as shown in more detail in Ref. 27 , provided all local-field effects ${ }^{28}$ are neglected.

\section{B. Lattice-induced excitation gaps in IXSS}

Intraband and interband transitions between oneelectron states, induced by the inelastic-scattering process, are represented by the RPA-equivalent selfconsistent expression for $\epsilon_{2}(\mathbf{q}, \omega) .{ }^{28}$ In an homogeneous electron system, the transitions for a given pair of $\mathbf{q}, \omega$ can be represented as occurring between planes in $\mathbf{k}$ space perpendicular to $q$ and separated by $q$, as shown in Fig. 1(a), where the corresponding $\epsilon_{2}(\mathbf{q}, \omega)$ is also sketched. Let one Fourier component $V(\mathbf{g})$ of the periodic crystal potential with $\mathbf{g} \| \mathbf{q}$ be switched on and let transitions end up on the g-related Bragg plane $\mathbf{k} \cdot \mathbf{q}=g^{2} / 2$. Then, due to the $V(\mathbf{g})$-induced energy gap, the energy transfer $\omega$, corresponding to this transition in the homogeneous case, cannot be realized in the $V(\mathbf{g})$ perturbed case, so that an excitation gap of width $2 V(\mathbf{g})$ occurs around

$$
\omega_{g}=(\hbar q / 2 m)(g-q)
$$

in $\epsilon_{2}(\mathbf{q}, \omega)$, as indicated in Fig. 1(b). Taking into account all other Fourier components of the crystal potential, (i) the excitation gap may get flattened to a certain extent, so that only a dip remains; (ii) the dip position may get shifted with respect to $\omega_{g}$ of Eq. (7) according to special features of the band structure. Flattening of the dip and shifting of the dip position also occur, when $q$ is tilted away from $g$.

According to

$$
\operatorname{Im} \epsilon^{-1}(\mathbf{q}, \omega)=-\epsilon_{2}(\mathbf{q}, \omega) /\left[\epsilon_{1}^{2}(\mathbf{q}, \omega)+\epsilon_{2}^{2}(\mathbf{q}, \omega)\right],
$$

lattice-induced dips for $\mathbf{q} \| \mathbf{g}$ should also be detected in $\operatorname{Im} \epsilon^{-1}$ and therefore also in $S(\mathrm{q}, \omega)$, where the $\operatorname{dip}$ position on the $\omega$ scale may be shifted a little bit with respect to the $\epsilon_{2}$-dip position due to the $\omega$ dependence of $\epsilon_{1}$. In special cases $\epsilon_{1}(\mathbf{q}, \omega)$ can exhibit an additional zero

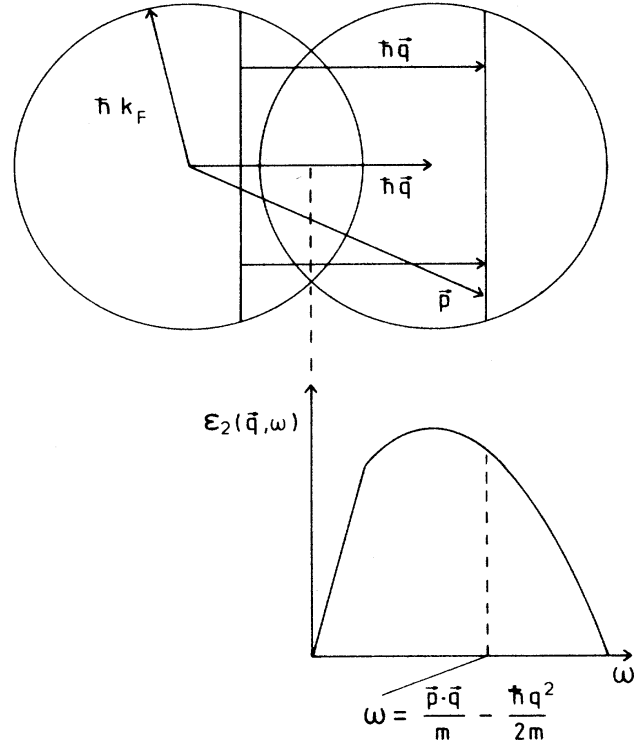

(a)

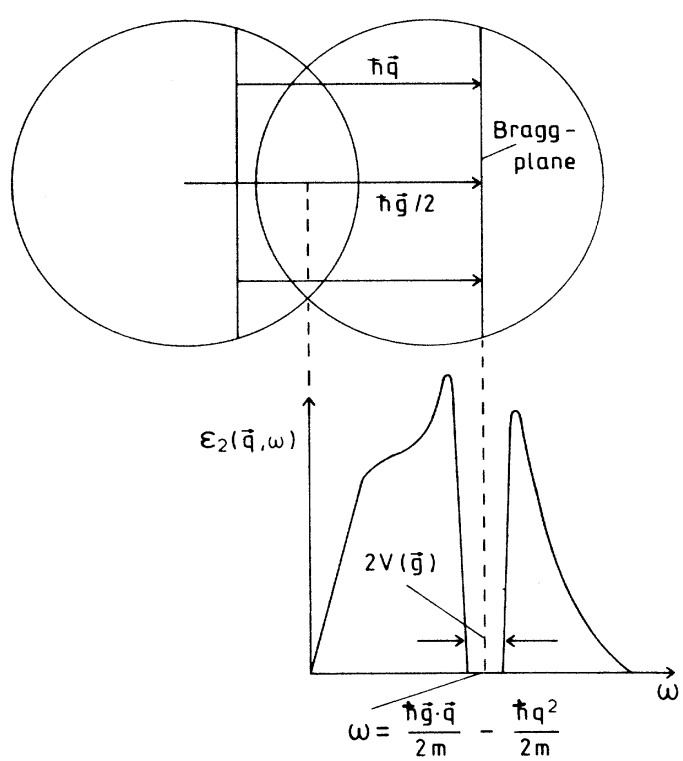

(b)

FIG. 1. (a) Upper part: Transitions between one-electron states in momentum space of an homogeneous electron system as connected with an inelastic-scattering process transferring momentum $\mathbf{q}$ and energy $\hbar \omega$. Lower part: Corresponding $\epsilon_{2}(\mathbf{q}, \omega)$. (b) Upper part: Transitions to a Bragg plane in the extended zone schemes of an inhomogeneous electron system. Lower part: Corresponding $\epsilon_{2}(\mathbf{q}, \omega)$ within the limits of a two-band approximation, exhibiting an excitation gap of width $2 V(\mathbf{g})$. 
within the dip, so that the condition for a collective excitation might be met (zone boundary collective state), as introduced by Foo and Hopfield ${ }^{29}$ and thoroughly discussed by Sturm and Oliveira. ${ }^{30}$

\section{Experiment}

The measurement of the DDCS was performed at the Hamburger Synchrotronstrahlungslabor using synchrotron $x$-rays from the storage ring DORIS. The experimental setup, consisting in a $\mathrm{Ge}(220)$-double-crystal monochromator and a $\mathrm{Si}(333)$ spherically bent crystal analyzer, has been described in more detail elsewhere. ${ }^{11,31}$ Utilizing dispersion compensation, ${ }^{11,32} 1.0 \mathrm{eV}$ overall energy resolution could be obtained with $6 \times 10^{9}$ useful photons of $5.95 \mathrm{keV}$ at the scattering sample. The sample crystal has been grown from $99.99 \%$ high-purity Be. The experiment has been performed in transmission geometry with $1.6-\mathrm{mm}$ sample thickness. The scattering sample was held in a scattering chamber evacuated to $10^{-5}$ Torr in order to prevent air scattering. $7 \times 10^{3}-1 \times 10^{4}$ counts were collected in a measuring point around the inelastically scattered peak. The Be data were processed in the same way as the $\mathrm{Li}$ data, ${ }^{11}$ including absolute scaling by utilizing the $f$ sum rule, so that the experimental results can be presented in the form of absolute values of the dynamic structure factor $S(\mathrm{q}, \omega)$.

\section{SUMMARY OF THE EXPERIMENTAL RESULTS}

In Fig. 2 the experimental dynamic structure factors of Be for $\mathbf{q}$ parallel to the most prominent reciprocal-lattice vectors and for $q$ values between $q=0.45$ a.u. and $q=1.69$ a.u. are plotted together with some results on polycrystalline samples. Thus $q$ covers a range between $0.66 q_{c}$ and $2.48 q_{c} \quad\left(q_{c}=\right.$ plasmon cutoff vector $=0.68$ a.u.). In Fig. 2 and in the following the direction of $q$ always is indicated in the form "q $\| h k l$," where $h k l$ refers to a direction in reciprocal space parallel to the reciprocallattice vector $\mathbf{g}_{h k l} \equiv h \mathbf{b}_{1}+k \mathbf{b}_{2}+l \mathbf{b}_{3} \quad\left(\mathbf{b}_{1}, \mathbf{b}_{2}, \mathbf{b}_{3}\right.$ basis vectors of the reciprocal lattice). The very strong qorientation dependence of the $S(\mathrm{q}, \omega)$ fine structure is striking: The differences between directions, which are only $30^{\circ}$ apart, amounts to more than $30 \%$ of the peak value. The lowest degree of fine structure is found in the q\|100 spectra, the highest for $q \| 110$. The 101 spectra exhibit the greatest similarity with the polycrystalline ones, where even the polycrystalline spectra are not completely free of fine structure: The shoulder in the $q=1.09$ a.u. and the $q=1.4$ a.u. polycrystalline spectra grows into a double-peak structure, which already has been noticed by other authors ${ }^{4,19}$ and has initiated a lot of theoretical work, as mentioned in the Introduction. The dispersion of the dip and peak positions in the $S(\mathbf{q}, \omega)$ fine structure is only weak but by far not negligible. In the following

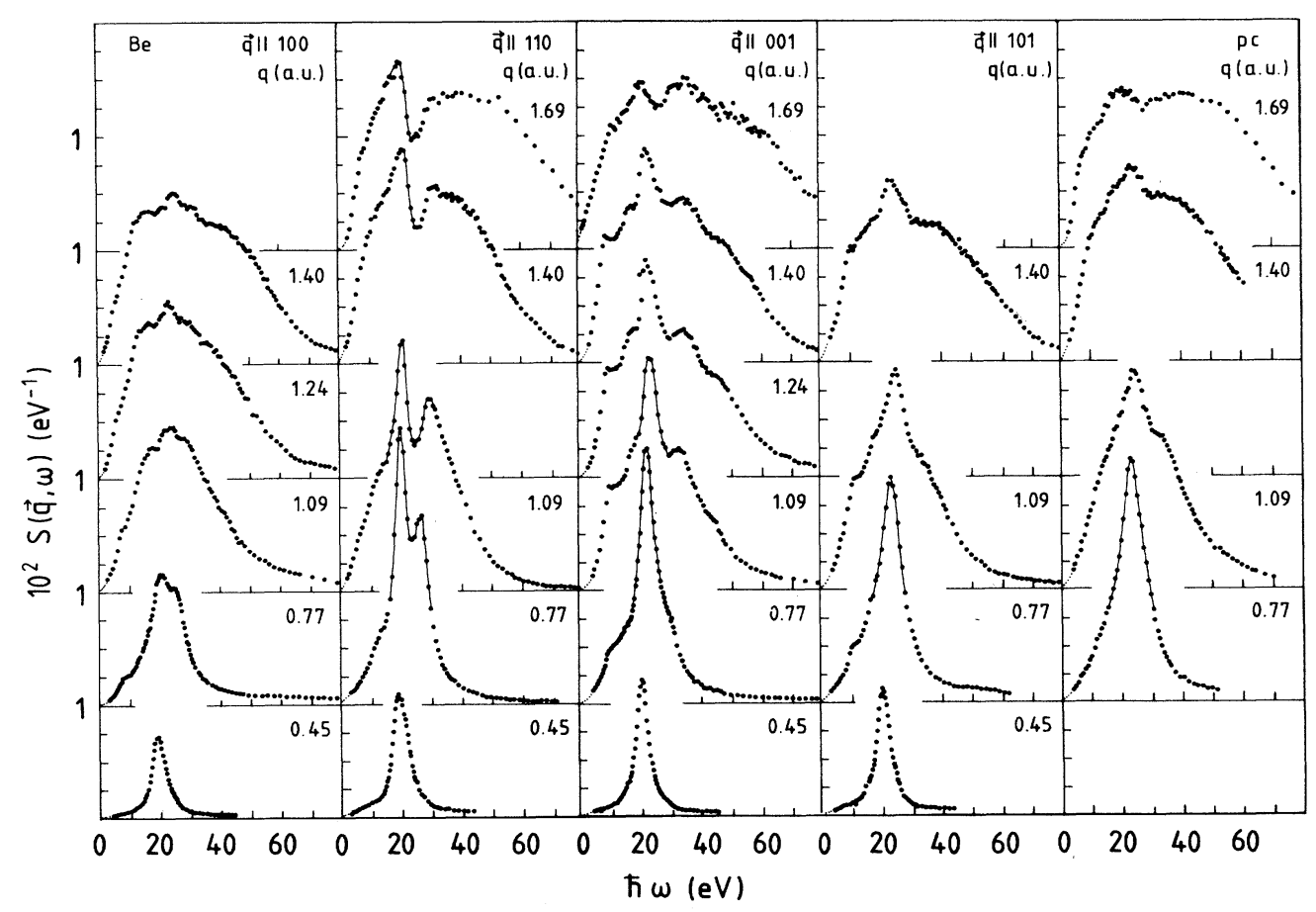

FIG. 2. Survey of the experimental dynamic structure factors $S(\mathbf{q}, \omega)$ of Be metal both from single crystals and from polycrystalline (pc) material for values and directions of $\mathbf{q}$ as indicated. The small dots in the region of small-energy transfer $\hbar \omega$ represent the less-reliable part of the spectra because of strong superposition by the Rayleigh line in the rough data. 
we will distinguish between two kinds of fine structure, one with very high q-orientation dependence, the other with much less sensitivity to variations of the $\mathbf{q}$ orientation. The very different q-orientation sensitivity is demonstrated by means of experiments, where the $\mathbf{q}$ direction has been changed in small steps leaving $q=1.09=$ const. The spectra of Fig. 3 have been obtained by tilting $\mathrm{q}$ from 001 through $90^{\circ}$ to 110 . The shoulder at roughly $12 \mathrm{eV}$ of the 001 spectrum disappears nearly completely by tilting $\mathbf{q}$ by less than $10^{\circ}$, whereas the double-peak structure with a dip roughly at $32 \mathrm{eV}$ withstands a tilting by more than $30^{\circ}$ without remarkable change either in shape or in position on the $\omega$ scale. Another example of a very orientation-sensitive fine structure of Fig. 3 is the double peak of the 110 spectrum, which is strongly shifted and degraded by tilting $\mathbf{q}$ by less than $15^{\circ}$. This strong orientation dependence of

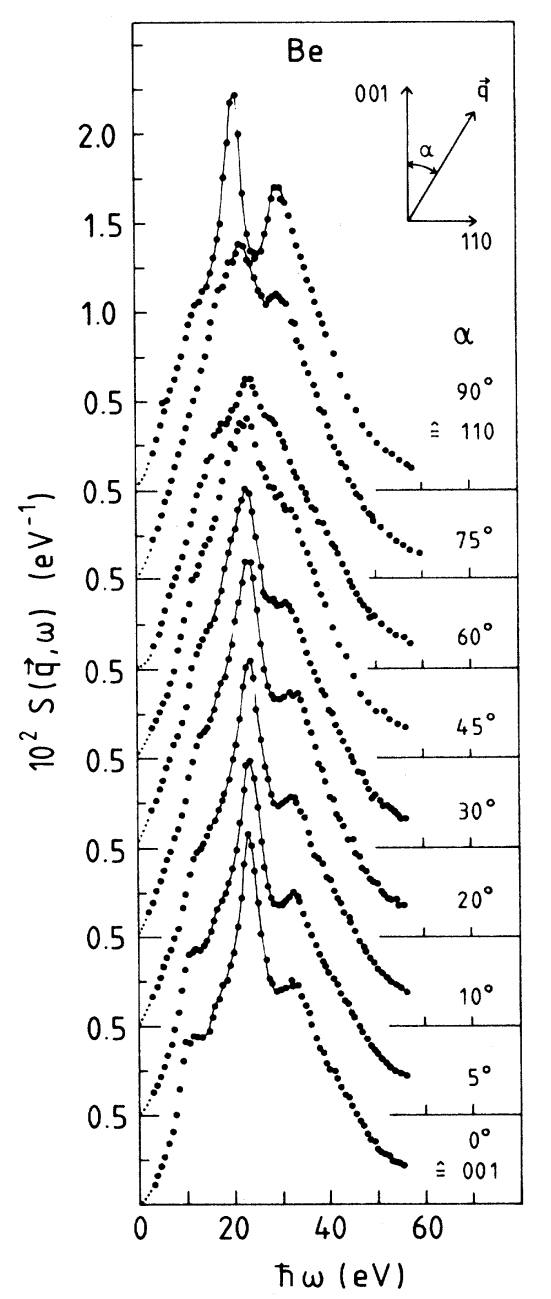

FIG. 3. Experimental dynamic structure factor $S(\mathbf{q}, \omega)$ of $\mathrm{Be}$ metal for $q=1.09$ a.u. obtained by tilting of $\mathbf{q}$ from $001(\alpha=0)$ into $110\left(\alpha=90^{\circ}\right)$. the 110-double-peak structure is again demonstrated in Fig. 4, where $q$ has been tilted by $30^{\circ}$ from 110 to 100 : Tilting of less than $20^{\circ}$ destroys the double-peak structure nearly completely. In Fig. 5 the result of rotating $q$ from 001 into 100 is displayed. Again the double-peak structure of the 001 orientation with a dip at $32 \mathrm{eV}$ is very stable against $q$ rotation, so that even a tilting of more than $40^{\circ}$ changes the double-peak structure only into a one-peak-one-shoulder structure, which persists till $60^{\circ}$. On the other hand, the fine structure between 10 and 20 $\mathrm{eV}$ changes very rapidly.

Finally, the influence of multiple-scattering events on the results could be estimated by measuring $S(\mathrm{q}, \omega)$ on samples of very different thickness $(1.5$ and $4.5 \mathrm{~mm}$, respectively), as shown in Fig. 6. If multiple scattering were of importance, the spectrum of the thicker sample should be distorted compared to that of the thinner sam-

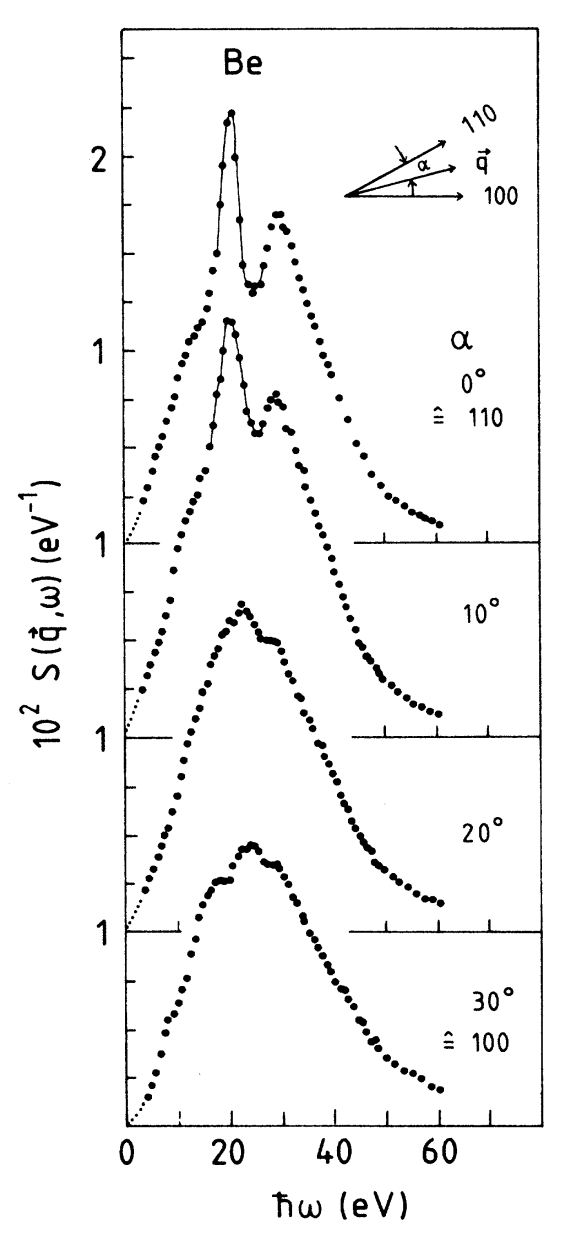

FIG. 4. Experimental dynamic structure factor $S(\mathrm{q}, \omega)$ of $\mathrm{Be}$ metal for $q=1.09$ a.u. obtained by tilting of $q$ from $110(\alpha=0)$ into $100\left(\alpha=30^{\circ}\right)$. 


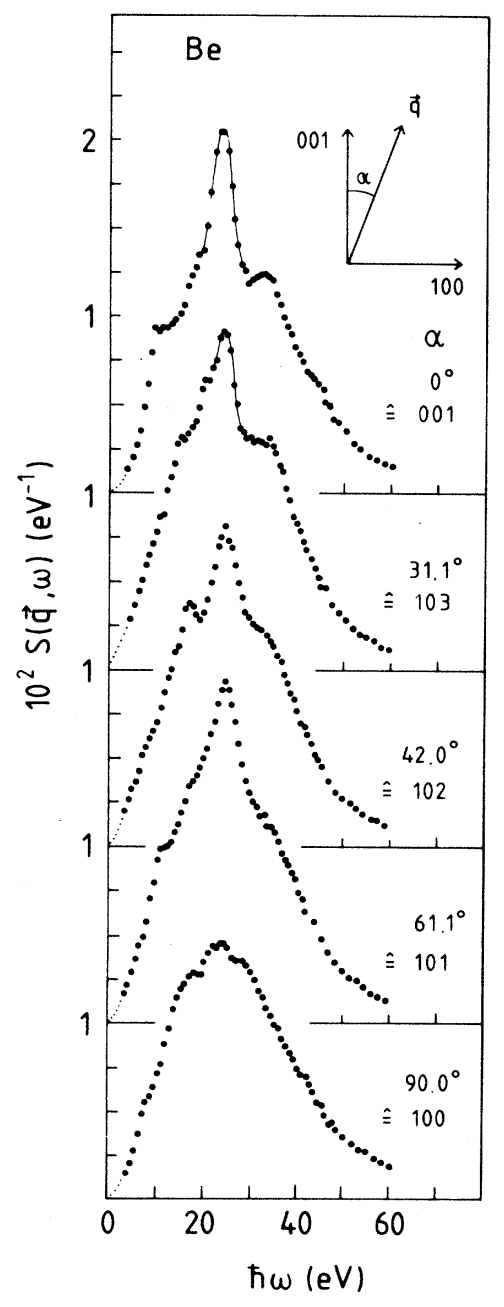

FIG. 5. Experimental dynamic structure factor $S(\mathbf{q}, \omega)$ of $\mathrm{Be}$ metal for $q=1.09$ a.u. obtained by tilting of $\mathbf{q}$ from $001(\alpha=0)$ into $100\left(\alpha=90^{\circ}\right)$ passing 103,102 , and 101.

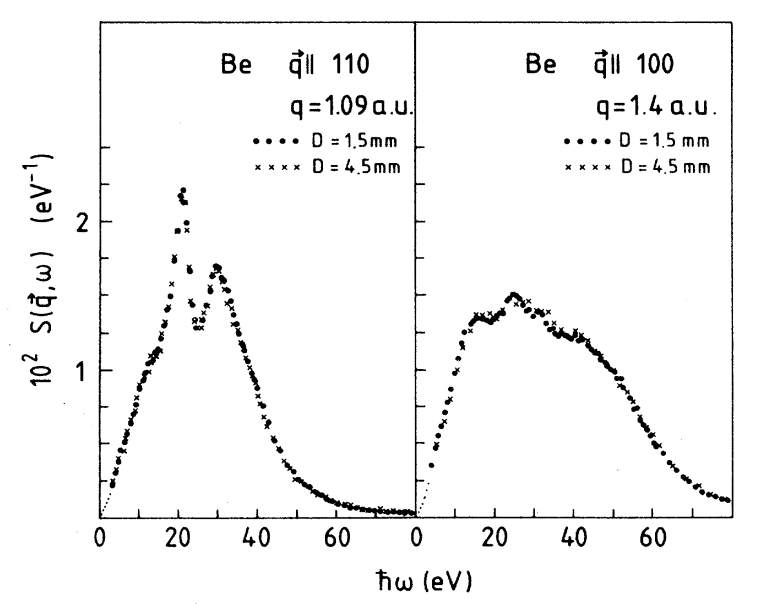

FIG. 6. Experimental dynamic structure factor $S(\mathbf{q}, \omega)$ of Be metal with $\mathbf{q} \| 110, q=1.09$ a.u., and $\mathbf{q} \| 100, q=1.4$ a.u., respectively, for two different sample thicknesses $D$ in order to demonstrate the rather small influence of multiple scattering. ple, since the probability of multiple-scattering events is increasing with sample thickness. We did not find any difference outside the statistical error (which was about $1 \%$ in Fig. 6 with the exception of the $100 ; D=4.5 \mathrm{~mm}$ spectrum, where the statistical error was much higher). Therefore, we conclude that multiple scattering at all is of minor influence on the final result.

\section{DISCUSSION OF THE RESULTS}

\section{A. Polycrystalline spectra versus model calculation for homogeneous systems}

The very strong $q$-orientation dependence of the $\operatorname{Be} S(q, \omega)$ fine structure, as shown in Sec. III, suggests its lattice origin. On the contrary, the overall shape of the spectra and possibly also the less-orientation-dependent fine structure should already be understood as due to properties of the homogeneous system. For example, in Ref. 19, the one-peak-one-shoulder fine structure, as seen even in the polycrystalline spectra, has been attributed to the momentum dependence of the quasiparticle lifetime. The most effective way to average over orientation-dependent properties in order to get the overall shape of the spectra in indeed to measure on polycrystalline samples. In doing so, of course, lattice effects do not have to be eliminated completely, so that the polycrystalline spectra will not necessarily solely reflect properties of the homogeneous system. Nevertheless, it seems justified to confront the polycrystalline results with $S(\mathrm{q}, \omega)$ spectra, which were calculated by using the following different models for the homogeneous system (described in more detail in Ref. 11):

(i) Random-phase approximation, taking into account damping within the limits of a simple relaxation-time approximation with $1 / \tau=0.03$ a.u., and by allowing for lattice effects only via the density of states effective mass of $\mathrm{Be}, m^{*}=1.13$, as calculated by $\mathrm{Shaw}^{33}$ at the Fermi level.

(ii) RPA with $m^{*}=1.13$, but replacing the Lindhard free-particle polarizability ${ }^{34}$ by an expression, first introduced by Mukhopadhyay, Kalia, and Singwi (MKS), ${ }^{12}$ which takes into account the momentum-dependent quasiparticle inverse lifetime $\Gamma(p)$ in a way shown in Eqs. (10) and (14) of Ref. 11. The inverse lifetime $\Gamma(p)$ has been calculated by means of Quinn's formalism ${ }^{35}$ by introducing higher-order excitations (both two-pair excitations and one-pair-plasmon excitations). Within the limits of Quinn's formalism we used the plasmon-pole model for the response function $\operatorname{Im}[1 / \epsilon(\mathbf{q}, \omega+i \delta(q)]$ first introduced by Lundquist, ${ }^{36}$ where the plasmon-dispersion law has been fitted to both the Be measurements of Eisenberger and Platzman ${ }^{5}$ and our Be data as shown in Fig. 2, so that the plasmon pole $\omega(q)$ follows the empirical equation

$$
\omega^{2}(q)=\left[\omega_{p}^{2}+\hbar^{2} k_{F}^{2} q^{2} / 3 m\right] f(q)+\left(\hbar q^{2} / 2 m\right)^{2},
$$

with

$$
f(q)=\left\{\begin{array}{l}
1 \text { for } q<q_{c} \\
\exp \left[-2\left(q-q_{c}\right)^{2}\right] \text { for } q>q_{c}
\end{array}\right.
$$


where $\omega_{p}$ is the free-electron plasmon frequency, $\hbar k_{F}$ is the Fermi momentum, and $q_{c}=0.66 \mathrm{a} . \mathrm{u}$. is the plasmon cutoff vector of Be. $\delta(q)$ (in a.u.) has been chosen in such a way that the widths of the experimental $S(\mathrm{q}, \omega)$ curves are reproduced:

$$
\begin{aligned}
\delta(q) & =\delta_{0}+0.115 q^{2} \text { for } q<q_{c}\left(\delta_{0}=0.06 \text { a.u. }\right) \\
& =0.43 q^{2} \text { for } q_{c}<q<1.5 k_{F} \\
& =q k_{F} / \sqrt{2} \text { for } q>1.5 k_{F} .
\end{aligned}
$$

The resulting inverse lifetime $\Gamma(p)$ is shown in Fig. 7 together with $\Gamma(p)$ as obtained by using the pure RPA expression for the response function. In both cases a steep rise of the inverse lifetime is found near a momentum $p_{0}$, where for $p>p_{0}$ the decay of a quasiparticle by emitting a plasmon becomes possible:

$$
\omega\left(q_{c}\right)=\hbar\left(p_{0}^{2}-k_{F}^{2}\right) \text {. }
$$

Therefore, in a very simple way $\Gamma(p)$ can be represented in a model with a step at $p_{0}$ (see Fig. 7).

(iii) Local-field- and lifetime-corrected scheme by using the MKS form of the free-particle polarizability of (ii), but by making additionally a local-field correction, first introduced by Hubbard ${ }^{37}$ [see Eq. (7) of Ref. 11]. The static approximation $G(q)$ of the local-field correction factor $G(q, \omega)$, as calculated by Utsumi and Ichimaru ${ }^{38}$ and shown in Fig. 8, has been utilized. The scheme (iii) for calculating $S(\mathbf{q}, \omega)$ might seem somewhat "ad hoc," but there exists a well-established theoretical foundation for proceeding this way. It was given within the framework of the so-called quasi-one-pair excitation approximation by Awa et al. ${ }^{14}$ The fundamental objection against this scheme, namely that it violates the conservation of local electron number, has been met both by Awa et $a l .{ }^{14}$ and by Niklasson et al. ${ }^{15}$ The authors state that the violation of the continuity equation is of minor importance, in so far as electron correlation in the inter-

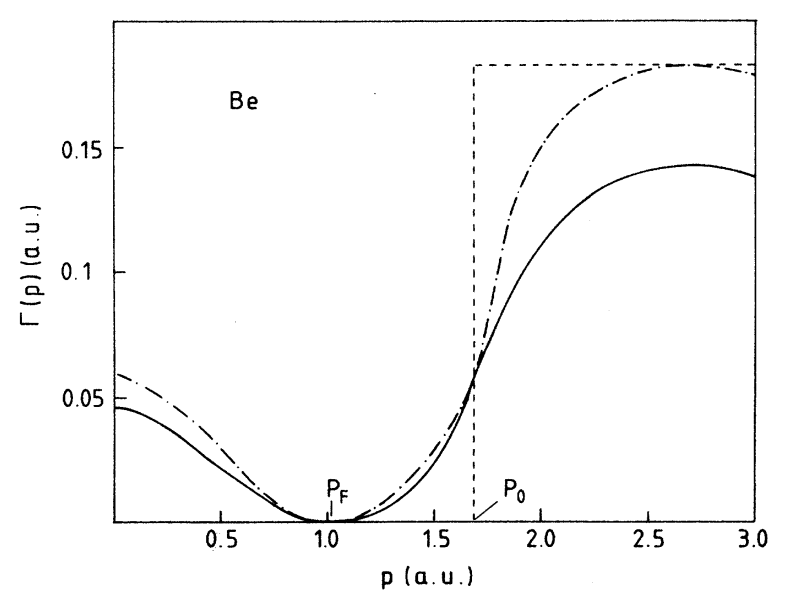

FIG. 7. Momentum-dependent inverse quasiparticle lifetime $\Gamma(p)$. Dashed line: step model, dashed-dotted line: RPA, solid line: plasmon-pole model on the basis of experimental data.

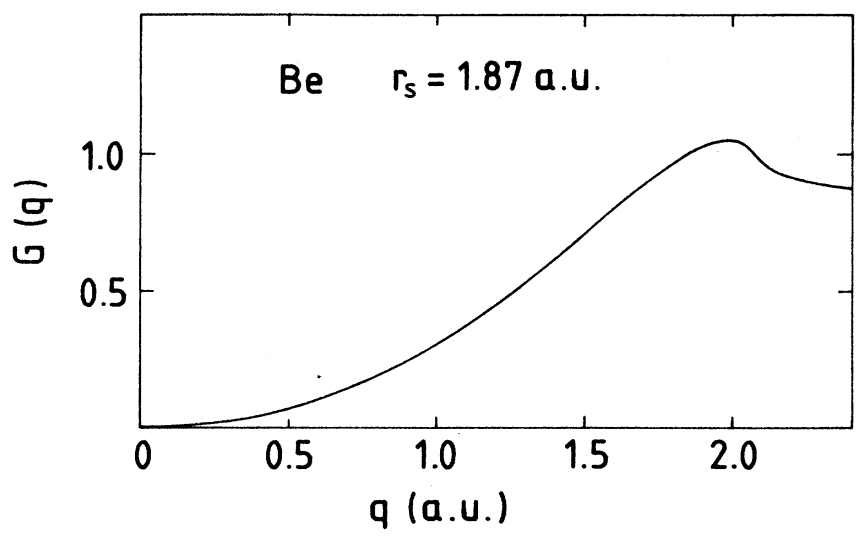

FIG. 8. Static local-field correction factor $G(q)$ for $r_{s}=1.87$ a.u. (Be) according to Ref. 38.

mediate wave number and frequency regime is concerned. Therefore, we feel justified to apply the scheme (iii) also to calculations of $S(q, \omega)$ within the limits of a two-band approximation, as shown later in Sec. IV B.

In Fig. 9 the results of the model calculations according to the three schemes (i) - (iii) has been confronted with the polycrystalline experiment. In order to facilitate the comparison, the experimental and calculated "peak positions" are listed in Table $I$. Because of the remaining fine structure of the experimental curves, the peak position is defined, in a somewhat arbitrary manner, to be the center of gravity at $\frac{2}{3}$ peak intensity, where we are well aware that this definition diminishes the differences between the various theoretical models because of the appreciable asymmetry of the theoretical curves. Nevertheless it becomes evident that by proceeding from model (i) to (iii) the agreement with experiment is improved for all values of $q$, where for small $q$ the experimental peak positions are at somewhat smaller, for larger $q$ at somewhat larger energies compared with model (iii). Therefore, it may be concluded that the simple RPA is far from reproducing the experimental overall shape of $S(\mathbf{q}, \omega)$. Only when momentum-dependent lifetime broadening together with local-field corrections are added, the main features of short-range correlation are represented appropriately, so that agreement between experiment and calculation becomes satisfactory. Small deviations to any side should not be overestimated in view of the remaining experimental fine structure.

TABLE I. Experimental (polycrystalline Be) and calculated "peak position" of $S(\mathrm{q}, \omega)$ in $\mathrm{eV}$ for different models.

\begin{tabular}{ccccc}
\hline & & $\begin{array}{c}\text { calc. } \\
\text { local-field } \\
\text { and lifetime } \\
\text { corr. }\end{array}$ & $\begin{array}{c}\text { lifetime } \\
\text { corr. }\end{array}$ & RPA \\
\hline (a.u.) & expt. & 23.6 & 24.7 & 25.7 \\
0.77 & 22.7 & 24.3 & 28.2 & 30.8 \\
1.09 & 25.2 & 27.4 & 32.4 & 35.6 \\
1.40 & 29.8 & 33.6 & 39.8 & 43.2 \\
1.69 & 36.9 & & & \\
\hline
\end{tabular}


One may wonder why in our calculations the momentum-dependent lifetime does not produce a distinct fine structure of $S(\mathbf{q}, \omega)$ in contrast to former calculations in Refs. 14-16. We have found that a fine structure, as came out in Refs. 14-16, can only be reproduced by using rather simple models for the momentumdependent lifetime. This is demonstrated in Fig. 10, where we found for $\mathrm{Be}$ with $q=1.69$ a.u. a distinct dip only by using the step model of Fig. 7. The RPA model produced a weak shoulder, whereas the plasmon-pole model, which takes into account realistic values for dispersion and broadening of the dielectric response, did not lead to any kind of fine structure. Moreover, it should be stressed that the step model-induced dip is in the wrong position by $8 \mathrm{eV}$, when compared with experiment. Therefore, the above comparison of model calculations with experiment does not support the assumption that the less-q-orientation-dependent part of the $S(\mathbf{q}, \omega)$ fine structure can be understood as due to the short-range correlations of the homogeneous system, so that a more detailed investigation about the origin of the $S(\mathrm{q}, \omega)$ fine structure is necessary and will be given in Secs. IV B and IV C. Nevertheless, we have not tested thoroughly whether introducing the frequency-dependent real part of the self-energy, as done by Niklasson, ${ }^{21}$ could produce a fine structure as found in the $p c$ experiment. We did not take into account the real part of the self-energy in all our calculations, since we felt that this real part is already contained in the local-field factor $G(q)$ in an average fashion, as already pointed out by Mukhopadhyay et al. ${ }^{12}$

\section{B. q-orientation-dependent fine structure versus model calculations}

By considering a two-band model, we have concluded in Sec. II B that excitation gaps due to the periodic potential of the electron-ion interaction should produce a diplike fine structure of $S(\mathbf{q}, \omega)$. A dip corresponding to a certain reciprocal-lattice vector $\mathbf{g}$ should become strongly visible whenever $\mathbf{q}$ coincides with the direction of $\mathbf{g}$, assuming the Fourier component $V(\mathbf{g})$ is different from zero. In order to prove this statement, we will confront experimental $S(\mathrm{q}, \omega)$ spectra having $\mathrm{q} \| 100,001$, $101,102,110$, and 103 with calculated spectra. The calculations were performed on the basis of a simple model band structure, where only those Fourier coefficients $V(\mathbf{q})$ of a local pseudopotential were taken into account, whose reciprocal-lattice vectors are both parallel or antiparallel to the $\mathbf{q}$ orientation of the corresponding experi-

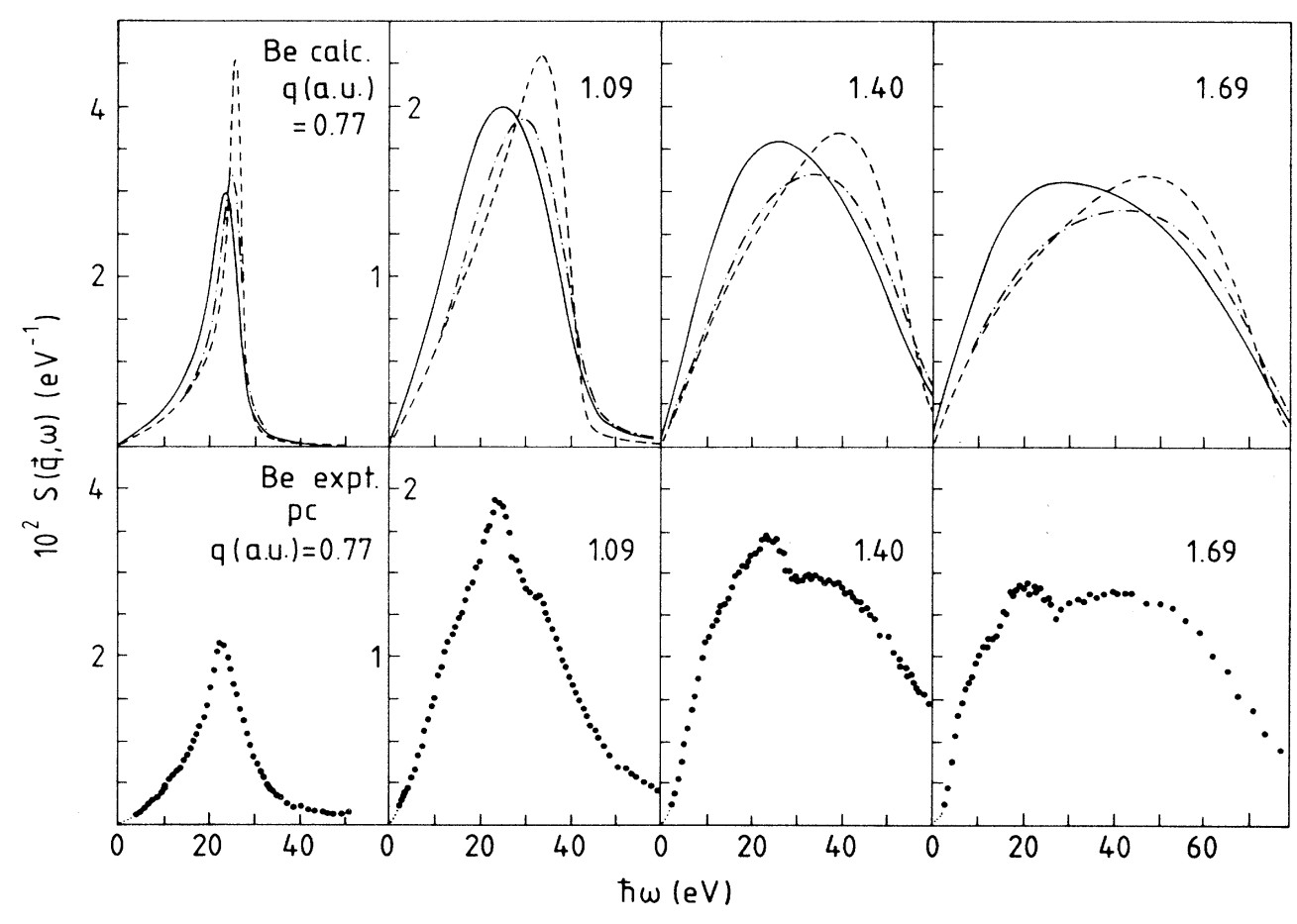

FIG. 9. Comparison of $S(\mathbf{q}, \omega)$ of polycrystalline Be metal (lower part) with theoretical $S(\mathbf{q}, \omega)$ (upper part) calculated according to different models of a homogeneous electron system of Be density and allowing for lattice effects by means of a density-of-state mass $m^{*}=1.13$. Dashed line: RPA, dashed-dotted line: RPA together with momentum-dependent quasiparticle lifetime, solid line: local-field-corrected and momentum-dependent quasiparticle lifetime. 


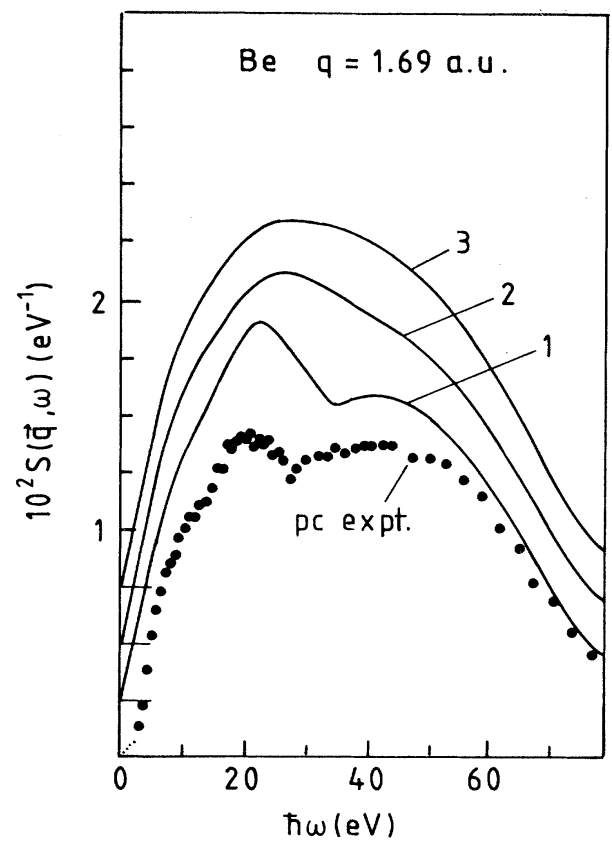

FIG. 10. Experimental polycrystalline $S(\mathbf{q}, \omega)$ of $\mathrm{Be}$ for $q=1.69$ a.u. confronted with calculated $S(\mathbf{q}, \omega)$, local-field corrected and with momentum-dependent lifetime according to the three models for $\Gamma(p)$ of Fig. 7. 1: step model, 2: RPA, 3: plasmon-pole model.

mental spectrum and do not have values larger than $2\left(q+k_{F}\right)\left(k_{F}=\right.$ Fermi momentum). The pseudopotential used is the local approximation of the nonlocal pseudopotential given in Eq. (2) of Ref. 39, where the lattice potential of Loucks and Cutler ${ }^{40}$ has been inserted. The nonlocal term of Eq. (2) of Ref. 39 has been evaluated with both states on the Fermi sphere of free-electron radius using free atomic Clementi-1s-core states. ${ }^{41}$ The following pseudopotential Fourier coefficients have been obtained (in a.u.): $V(100)=0.094 ; V(002)=.156 ; V(101)=.124$; $V(102)=0.04 ; \quad V(110)=0.06 ; \quad V(103)=0.047 ; \quad V(200)$ $=0.026 ; V(004)=0.048$. The further theoretical treatment of $S(\mathbf{q}, \omega)$ followed the scheme (iii) of Sec. IV A (local-field and lifetime corrections), also allowing for the nonlocal character of the pseudopotential by means of a density of states mass $m^{*}=1.13 .{ }^{33}$ [With one exception, the above-given Fourier coefficients were large enough to reproduce a diplike structure of equal or even larger size than that found in the experiment. Only for $q \| 103$, $V(103)$ had to be set at 0.085 a.u. to better fit the experiment.] In Figs. 11 and 12, experimental and modelcalculated dynamical structure factors are confronted for different directions of q. Dip-type fine structures, which belong together, can be identified and are marked by arrows (broken arrows in cases where the fine structure is less distinct in the spectra). In order to get a more quantitative comparison as far as the dip position is concerned, the third derivative of $S(\mathbf{q}, \omega)$ is plotted in Figs. 11 and 12. The dip position, as listed in Table II, is defined as the zero of the third derivative. [The definition

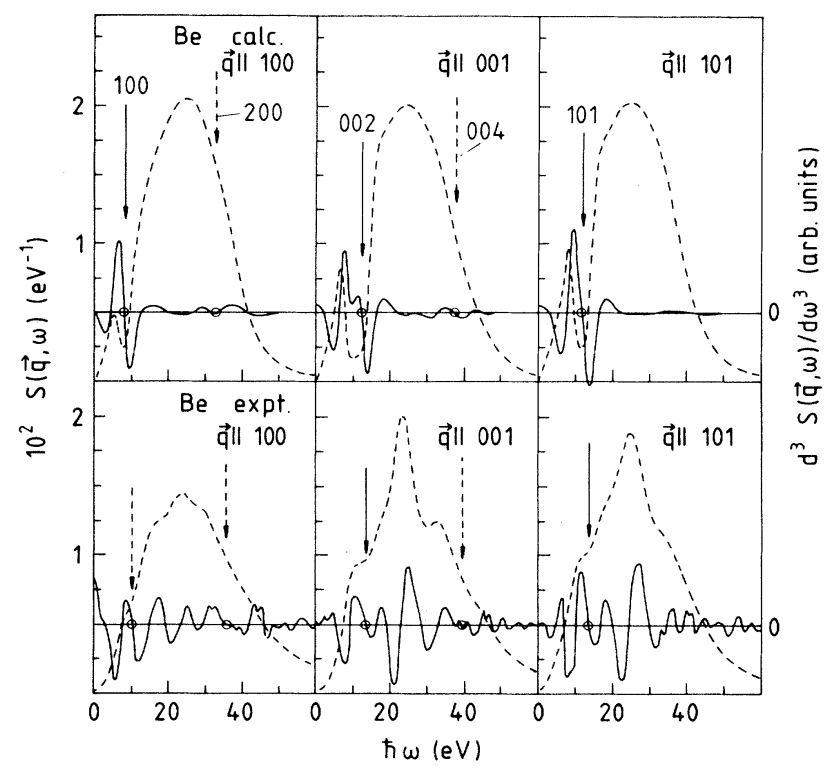

FIG. 11. Lower part: Smoothed experimental $S(\mathbf{q}, \omega)$ (dashed line); third derivative of experimental $S(q, \omega)$ (solid line) for $\mathbf{q} \| 100,001$, and 101. Upper part: Model calculated $S(\mathbf{q}, \omega)$ (dashed line); third derivative of calculated $S(\mathbf{q}, \omega)$ (solid line). Solid arrows are pointing to excitation-gap-induced dips (zeros of the third derivative), indexing of the arrows according to the Bragg-plane involved; dashed arrows indicate less-pronounced dips.

is correct when the dip can be represented as an inverse Lorentzian superimposing a section of a free electron $S(\mathbf{q}, \omega)$, where the section can be approximated by a parabola.] Summarizing, it can be stated that for all reciprocal-lattice vectors from $\mathbf{g}_{100}$ to $\mathbf{g}_{103}$, an excitation-

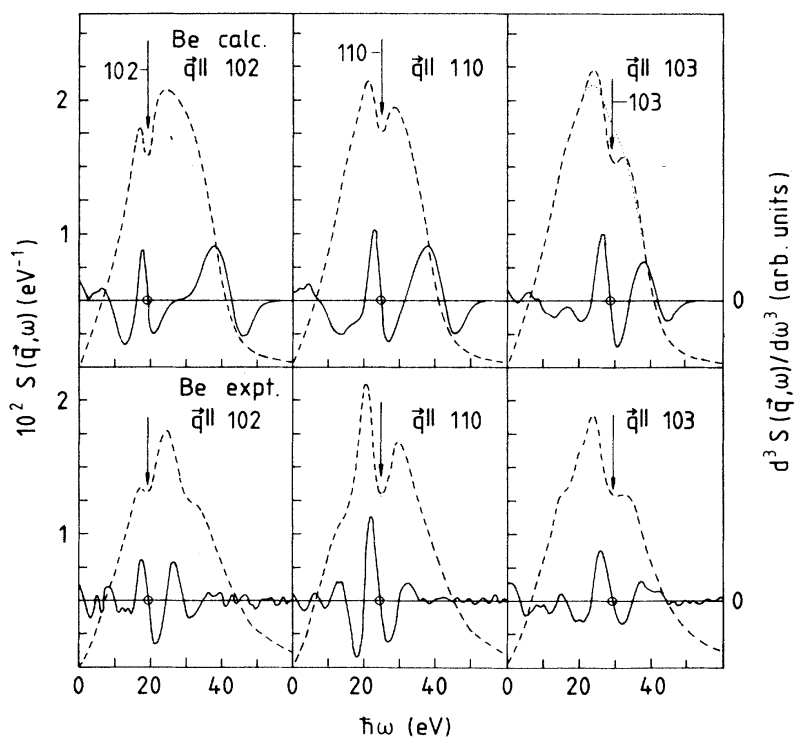

FIG. 12. Same as Fig. 10, but for q\|102, 110, and 103. 
TABLE II. Experimental and model calculated dip position of $S(\mathbf{q}, \omega)$ in eV for different orientations of $\mathbf{q}$ and related to Bragg planes as indicated.

\begin{tabular}{cccr}
\hline \hline q orientation & Bragg plane & expt. & calc. \\
\hline 100 & 100 & $10.2 \pm 1.0$ & 8.1 \\
& 200 & $35.4 \pm 2.0$ & 33.4 \\
001 & 002 & $13.8 \pm 0.5$ & 11.9 \\
& 004 & $37.6 \pm 2.0$ & 38.0 \\
101 & 101 & $13.6 \pm 1.0$ & 12.7 \\
102 & 102 & $19.5 \pm 0.5$ & 19.5 \\
110 & 110 & $24.6 \pm 0.5$ & 24.8 \\
103 & 103 & $29.1 \pm 1.0$ & 29.6 \\
\hline \hline
\end{tabular}

gap-induced diplike fine structure can be identified. The dip is well defined in the experimental curves and is found within less than $0.5 \mathrm{eV}$ at the calculated position in those cases where both the corresponding Bragg plane is completely surrounded by unoccupied $\mathbf{k}$-space of the extended zone scheme, and the final states are within the lowdamped region of Fig. 7. The excitation-gap-induced fine structure of the experimental curves is reduced to a shoulder, and its "dip position," according to the above definition, is found at larger $\hbar \omega$ compared to the calculation by up to $2 \mathrm{eV}$ in those cases, where the corresponding Bragg plane bounds occupied $\mathbf{k}$ space of the extended zone scheme $\left(\mathbf{g}_{100}, \mathbf{g}_{002}\right.$, and $\left.\mathbf{g}_{101}\right)$. In the latter case, details of the band structure near the Fermi level seem to be much more decisive, so that a simple two-band model cannot fully account for the lattice-induced fine structure. Within the region of strong damping of the final states $\left(\mathbf{g}_{200}\right.$ and $\left.\mathbf{g}_{004}\right)$, both the experimental and the theoretical fine structure are washed out nearly completely. Another verification that lattice-induced excitation gaps are the origin of the diplike fine structure has been given in Ref. 20 by investigating the dispersion of the dip position both in experiment and in model calculations.

Assuming the interpretation of the fine structure as given above is correct, we can utilize the dispersion of the dip position to probe the occupied part of the band structure $E(\mathbf{k})$ being guided by the following consideration: Let $\mathbf{q}$ be parallel to a certain reciprocal-lattice vector $\mathbf{g}$. Then the dip position $\hbar \omega_{d}(\mathbf{q})$ on curves of $\epsilon_{2}(\mathbf{q}, \omega)$, which were obtained by Kramers-Kronig analysis of the experimental $S(\mathbf{q}, \omega)$, corresponds to final states with wave vector $\mathbf{k}^{\prime}$ and energy $E\left(\mathbf{k}^{\prime}\right)$ on Bragg planes in the extended zone scheme, given by $\mathbf{k}^{\prime} \cdot \mathbf{g}=g^{2} / 2$. The corresponding initial states are characterized by a wave vector $\mathbf{k}$ given by

$$
\mathbf{k}=\mathbf{k}^{\prime}-\mathbf{q}
$$

whose energy is connected with the energy transfer $\hbar \omega_{d}(\mathbf{q})$ via

$$
E(\mathbf{k})=E\left(\mathbf{k}^{\prime}\right)-\hbar \omega_{d}(\mathbf{q}) \text {. }
$$

Therefore, by changing $q$ the dispersion of the occupied energy bands $E(\mathbf{k})$ can be observed relative to the energy $E\left(\mathbf{k}^{\prime}\right)$, where $\mathbf{k}^{\prime}$ is fixed to the Bragg plane. This bandstructure information is averaged over a plane in $\mathbf{k}$ space given by

$$
k_{z} g \equiv \mathbf{k} \cdot \mathbf{g}=g(g / 2-q),
$$

where each $\mathbf{k}$ is connected with the corresponding $\mathbf{k}^{\prime}$ via Eq. (12). Therefore, mainly the gross features of the band structure, e.g., the exchange-correlation induced bandwidth, ${ }^{42}$ can be probed and compared with theoretical calculations. This aspect of IXSS measurements has not yet been investigated systematically. Only the $110 \mathrm{spec}-$ tra have been evaluated that way in a rather preliminary manner. In Fig. 13 the experimental $110-\epsilon_{2}(\mathbf{q}, \omega)$ curves are plotted. The dip position (zero of the third derivative of $\epsilon_{2}$ ) is marked by an arrow. The band dispersion can directly be seen by the movement of the arrows relative to the broken line, which indicates roughly the $k_{z}=0$ position. In Fig. 14 the dip position $\hbar \omega_{d}$ is plotted relative to $\hbar \omega_{0} \equiv \hbar \omega_{d}(q=1.4$ a.u. $)$ as a function of $k_{z}$. The experimental dispersion $\left[\hbar \omega_{0}-\hbar \omega_{d}(\mathbf{q})\right]$ has been compared both with a free-electron parabola and with bandstructure calculations of Refs. 43 and 44, whereby $k_{z}=0$

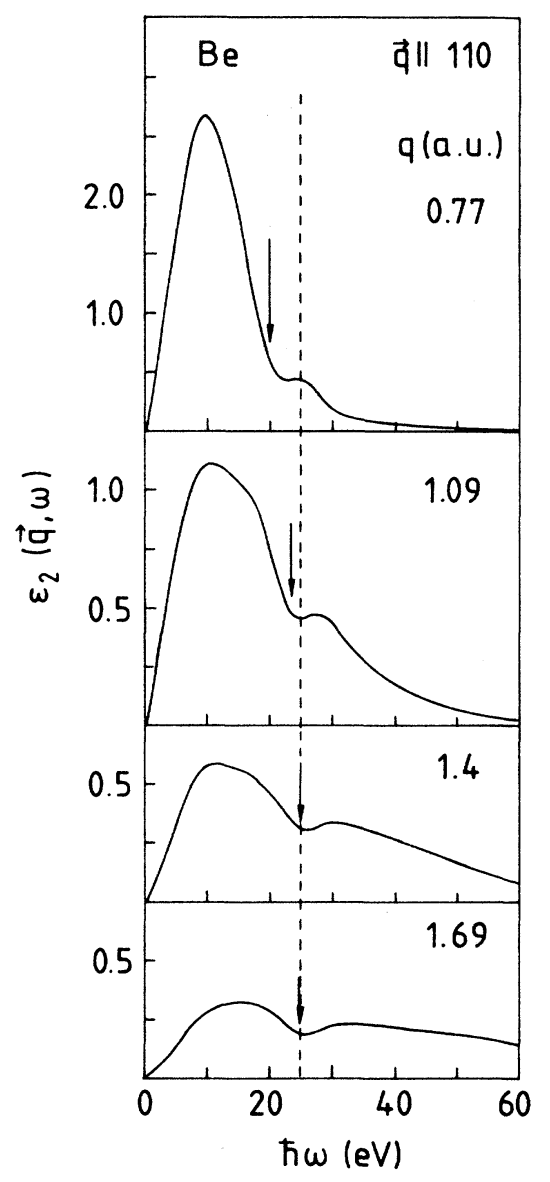

FIG. 13. Experimental $\epsilon_{2}(\mathbf{q}, \omega)$ [obtained by Kramers-Kronig transformation of $S(q, \omega)]$ of Be for $q \| 110$ and different values of $q$. Dip position (zero of the third derivative of $\epsilon_{2}$ ) is marked by an arrow. Dashed line indicates $\hbar \omega_{0}$. 


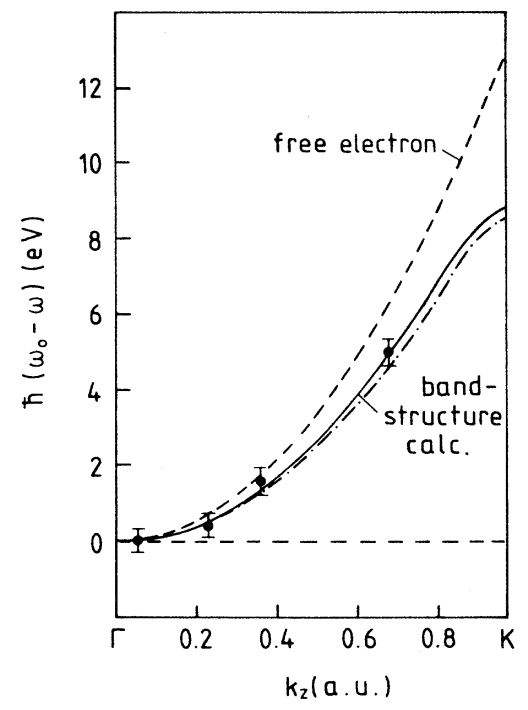

FIG. 14. Points: experimental dispersion of the relative dip position as a function of $k_{z}$. Lines: calculated $\Gamma-K$ bands by identifying $k_{z}=0$ with the $\Gamma$ point. Solid line: Ref. 44. Dotted-dashed line: Ref. 43. Dashed lines: free electron.

has been identified with the $\Gamma$ point. The experimental data of Fig. 14 are still too poor either to settle on a particular band-structure calculation or to estimate the amount of self-energy corrections to the band structure. ${ }^{42}$
Nevertheless, both sufficient statistical accuracy and a narrow mesh of $q$ values seems to be achievable, so that sensitive tests of band-structure calculations could be performed, especially if the averaging over planes in $\mathbf{k}$ space is already included in the band-structure calculated data.

\section{Discussion of the less-q-orientation-dependent fine structure}

All fine structure of $S(\mathbf{q}, \omega)$ discussed so far in Sec. IV B exhibits a strong q-orientation dependence. The prototype of a less q-orientation-dependent fine structure, the dip of the 001 spectra near $32 \mathrm{eV}$ for $q=1.09$ a.u., which withstands tilting by more than $40^{\circ}$ from 001 , so that it even persists in polycrystalline samples, shall now be discussed. There exist the following three indications that also this kind of fine structure could be lattice induced, though not in a simple manner, as discussed for structures in Sec. IV B, since it cannot be attributed to an excitation gap of a single Bragg-plane:

(i) The "32-eV" fine structure disappears for certain directions of $q$, e.g., for $q \| 100$.

(ii) The "32-eV" fine structure is reproduced in the only full band-structure calculation of $\mathrm{Be}-S(\mathbf{q}, \omega)$ for q\|001, performed by Taut and Hanke, ${ }^{23}$ as demonstrated in Fig. 15, where the calculated $S(\mathbf{q}, \omega)$ curves (normalized to the experimental peaks) are confronted with corresponding measurements of nearly equal $q$ values. At first sight, the theoretical and experimental curves seem

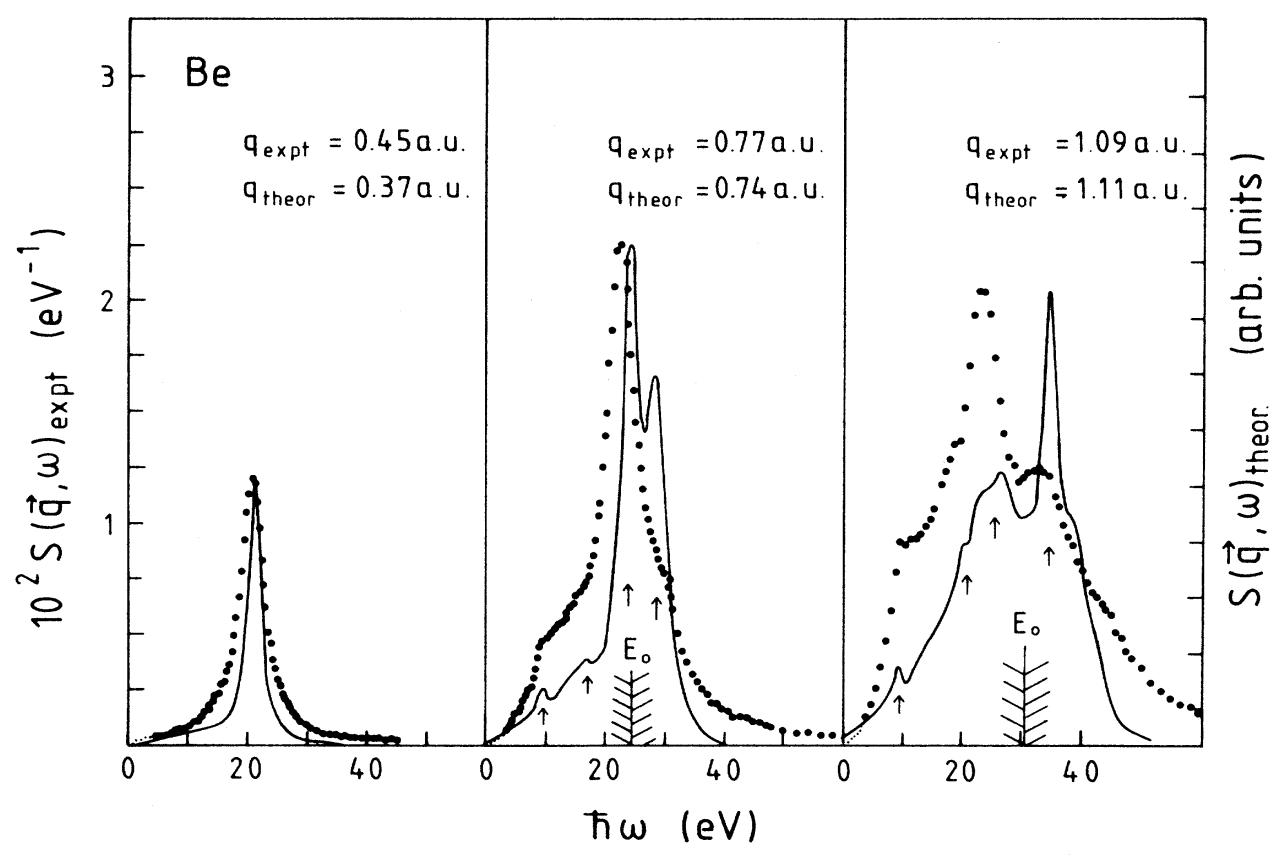

FIG. 15. Points: experimental $S(\mathbf{q}, \omega)$ of Be for $\mathbf{q} \| 001$ and $q_{\text {exp }}$ values as indicated. Solid line: calculated $S(\mathbf{q}, \omega)$ of Ref. 23 normalized to the experimental peaks. The energy transfer $\hbar \omega=E_{0}$ corresponds to the steplike rise of the reciprocal lifetime at $p_{0}$ as shown in Fig. 7. 
to be very different. But it has to be kept in mind that the calculations of Ref. 23 have been performed without taking into account the state-dependent lifetime (imaginary part of the self-energy) of excited quasiparticles as done in our calculations. If the authors of Ref. 23 had used the step model for the reciprocal lifetime of Fig. 7, that part of the calculated $S(\mathbf{q}, \omega)$ with $\hbar \omega>E_{0}$, where

$$
E_{0}=\left(\hbar q / m^{*}\right)\left(p_{0}-\hbar q / 2\right)
$$

and $p_{0}$ is defined by Eq. (11), would have been strongly broadened, whereas the part with $\hbar \omega<E_{0}$ would be left much less broadened, so that the intensity ratio of the peaks on both sides of $E_{0}$ for $q=1.09 / 1.11 \mathrm{a} . \mathrm{u}$. possibly would have become reversed. When comparing experiment and calculation in Fig. 15, the one-to-one correspondence of all peaks (indicated by arrows) is remarkable, although small deviations of their positions on the $\omega$ scale have to be admitted, possibly due to correlation-induced variations of the bandwidths. It should be stressed that the full band-structure calculation

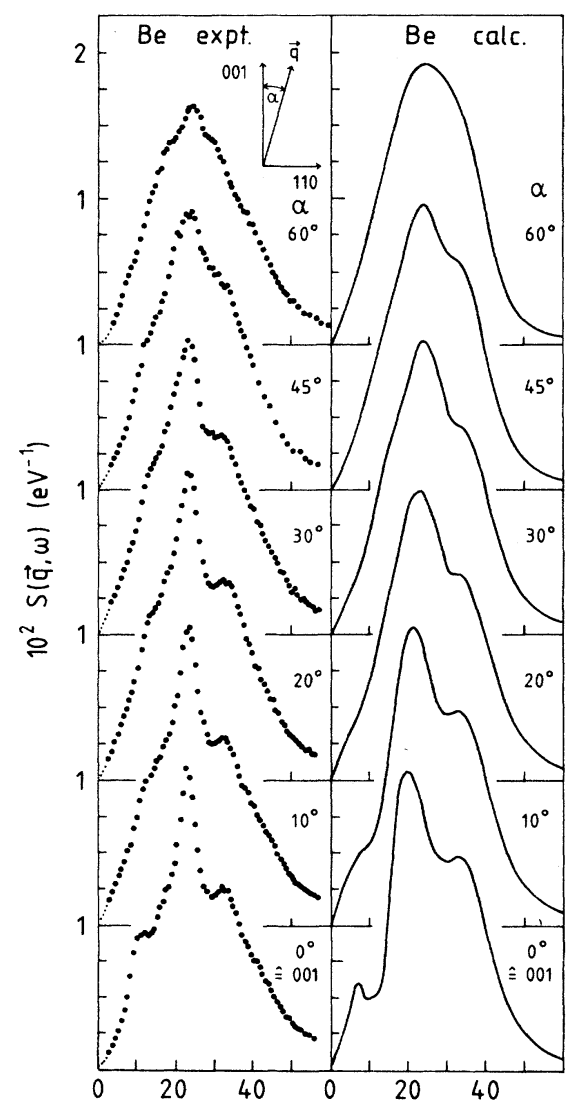

FIG. 16. Experimental (points) and model-calculated (solid line) q-orientation dependence of $S(\mathbf{q}, \omega)$ of Be for $q=1.09$ a.u. $\alpha$ is the angle of $q$ with $001 . V_{0}(103)=0.18$ a.u.; $V_{0}(002)=0.16$ a.u.; $\sigma(103)=0.4$ a.u.; $\sigma(002)=0.83$ a.u. even reproduces a fine structure, which has been attributed to an excitation gap due to the 002 Bragg plane.

(iii) Both the position of the "32-eV" fine structure and its stability against the variation of $q$ orientation can be reproduced by means of the following relatively simple model calculation, which possibly makes the physical origin of this fine structure more transparent: Because the position of the " $32-\mathrm{eV}$ " dip is not far from the $103 \mathrm{dip}$, which has been found for $q \| 103$, it can be assumed that the "32-eV" dip for q\|001 is due to an excitation gas as a consequence of the collective action of those 103-type Bragg planes, which are cutting $\mathbf{g}_{002}$ in one point. In order to prove this assumption in a physically transparent manner, we used, in principle, the same simple model calculation as in Sec. IV B. For this reason we divided the whole $\mathbf{k}$ space of the extended zone scheme into six prisms, each parallel to $q$ and each associated with one of the above-mentioned 103-type Bragg planes. Each prism was defined in such a way that points on its sectional plane with the associated 103-type Bragg plane have a shorter perpendicular distance to the corresponding 103type reciprocal-lattice vector than points on its sectional plane with other 103-type Bragg planes to the corresponding 103-type reciprocal-lattice vectors. The contri-

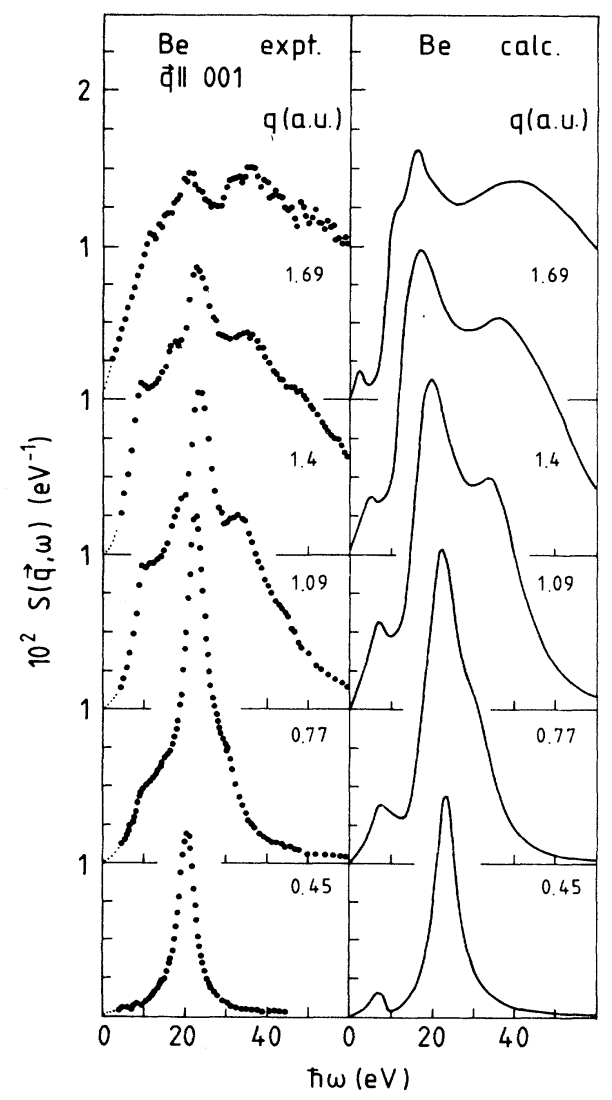

FIG. 17. Experimental (points) and model-calculated (solid line) $q$ dispersion of $S(\mathbf{q}, \omega)$ of Be for $\mathbf{q} \| 001$. Model parameters same as in Fig. 16. 
butions to $S(\mathrm{q}, \omega)$ of transitions between states within one prism were again calculated within the limits of a simple model band structure, where only those Fourier coefficients $V(\mathbf{g})$ of a local pseudopotential were taken into account, which correspond either to $\pm g_{1}$, where $g_{1}$ is a 103-type reciprocal-lattice vector, associated with the prism, or to a reciprocal-lattice vector $\mathbf{g}_{2}$, whose orientation is nearly parallel or antiparallel to $\mathbf{q}$. In contrast to the model calculations of Sec. IV B, we simulated additionally a variation of the band-gap width on the Braggplane by assuming $V(\mathbf{g})$ to vary as

$$
V\left(\mathbf{g}_{1 / 2}\right)=V_{0}\left(\mathbf{g}_{1 / 2}\right) \exp \left(-k_{1}^{2} / 2 \sigma_{1 / 2}^{2}\right),
$$

with $\mathbf{k}_{1} \cdot \mathbf{g}_{1 / 2}=0$. For these model calculations, $V_{0}(\mathbf{g})$ and $\sigma$ serve as free parameters and are chosen in Figs. 16 and 17 to fit the $S(\mathrm{q}, \omega)$ fine structure of the spectrum with q\|001, $q=1.09$ a.u. $\left(\alpha=0^{\circ}\right)$. Therefore, since we have fitted only one spectrum, the variation of the spectra both with $q$ orientation (see Fig. 16) and with $q$ values (see Fig. 17) are not much influenced by parametrizing. Moreover, it should be emphasized that in any case the position of the calculated fine structure is mainly determined by $m^{*}$, which has been taken from the first-principle calculations. $^{33}$

Comparison of the calculated q-orientation dependence of Fig. 16 with experiment leads to the conclusion that the model produces roughly the same stability of the "32-eV" dip against variations of the q orientation as seen in the experiment, whereas the dip around $12 \mathrm{eV}$, which we attributed to the 002 Bragg plane, is very strongly q-orientation dependent both in experiment and calculation.

Also, the model calculated dispersion of the less-qorientation-dependent fine structure, as shown in Fig. 17, is in fairly good agreement with experiment.

Therefore, it seems to be rather probable that also the less-q-orientation-dependent fine structure of $S(q, \omega)$ can be attributed to lattice effects (electron-ion interaction). This statement also applies to the polycrystalline sample, since the solid angle spanned by the $\mathbf{q}$ orientation with stable " $32-\mathrm{eV}$ " fine structure is roughly $2.5 \pi$.

On the one hand, it cannot be excluded completely that at least part of the less-q-orientation-dependent fine structure is due to special features of electron-electron interaction, where, in the case of the 100 and 110 spectra, such a fine structure had to be superimposed completely by a strong lattice-induced structure. Such an interpretation might be supported by the fact that we needed in Sec. IV B a 103 Fourier coefficient of the pseudopotential, which had to be by $100 \%$ larger than the calculated one, in order to account for the q\|103 dip, whereas all other
Fourier coefficients were large enough to reproduce the experimental fine structure.

But, on the other hand, the most plausible explanation of the q-orientation-independent fine structure on the basis of electron-electron interaction proposed so far, namely the introduction of higher order (two-pair and one-pair-one-plasmon excitations) via the quasiparticle lifetime, did not reproduce this fine structure either at the right position or with the correct strength when calculations were done in a realistic manner, as shown in Sec. IV A. For reasons we have already mentioned in Sec. IV A, we have not tested whether the frequency dependence of the real part of the self-energy can account for a q-orientation-independent fine structure at a position and with a strength we have found experimentally. The calculation of Niklasson ${ }^{21}$ does not seem to be very encouraging in this respect.

\section{CONCLUSIONS}

The comparison of experimental dynamic structure factors $S(\mathbf{q}, \omega)$ of single crystalline and polycrystalline $\mathrm{Be}$ metal over a wide range of $q$ with model calculated ones have lead to the following conclusions.

(i) A mean field theory such as the RPA, together with restriction to single excitations, cannot account appropriately for short-range electron correlation.

(ii) Only when taking into account both short-range fluctuations (local-field corrections) and multiple excitations via a state-dependent quasiparticle lifetime, the short-range electron correlations can be allowed for in such a way that the overall features of the dynamical structure factor are correctly represented.

(iii) The fine structure of the dynamical structure factor, in so far it is strongly q-orientation dependent, could be attributed to excitation gaps as due to Bragg planes of the extended zone scheme. Therefore, in principle, the fine structure of $S(\mathrm{q}, \omega)$ can be utilized for band-structure probing.

(iv) There exist strong indications that even the less-qorientation-dependent fine structure of $S(\mathbf{q}, \omega)$ has to be attributed to ion-electron interaction, although an electron-correlation-induced origin cannot be completely ruled out.

We thank U. Bonse, K. Fischer, R. Nusshardt, A. Millhouse, and H. Schenk-Strauss for valuable help with the measurements at Doppel-Ring Speicheranlage (DORIS), Hamburger Synchrotronstrahlungslabor (DESY). This work has been funded by the German Federal Minister of Research and Technology under Contract No. 05-334-AX-B2.
${ }^{1}$ For a recent review see W. Schülke, Nucl. Instrum. Methods A 280, 338 (1989).

${ }^{2}$ P. Eisenberger, P. M. Platzman, and K. C. Pandy, Phys. Rev. Lett. 31, 311 (1973).

${ }^{3}$ P. Eisenberger, P. M. Platzman, and P. Schmidt, Phys. Rev. Lett. 34, 18 (1974).

${ }^{4}$ P. M. Platzman and P. Eisenberger, Phys. Rev. Lett. 33, 152
(1974).

${ }^{5}$ P. Eisenberger and P. M. Platzman, Phys. Rev. B 13, 934 (1976).

${ }^{6}$ P. Vashishta and K. S. Singwi, Phys. Rev. B 6, 875 (1972).

${ }^{7}$ E. Pajanne, J. Phys. C 15, 5629 (1982).

${ }^{8}$ K. Utsumi and S. Ichimaru, Phys. Rev. B 22, 1522 (1980); 22, 5203 (1980); 23, 3291 (1981). 
${ }^{9}$ H. De Raedt and B. De Raedt, Phys. Rev. B 18, 2039 (1978).

${ }^{10}$ F. Green, D. Neilson, and J. Szymansky, Phys. Rev. B 31, 2779 (1985); 31, 2796 (1985); 31, 5837 (1985).

${ }^{11}$ W. Schülke, H. Nagasawa, S. Mourikis, and P. Lanzki, Phys. Rev. B 33, 6744 (1986).

${ }^{12}$ G. Mukhopadhyay, R. K. Kalia, and K. S. Singwi, Phys. Rev. Lett. 34, 950 (1975).

${ }^{13}$ G. Mukhopadhyay and A. Sjölander, Phys. Rev. B 17, 3589 (1978).

${ }^{14}$ K. Awa, H. Yasuhara, and T. Asahi, Phys. Rev. B 25, 3670 (1982); 25, 3687 (1982).

${ }^{15}$ G. Niklasson, A. Sjölander, and F. Yoshida, J. Phys. Soc. Jpn. 52, 2140 (1983).

${ }^{16}$ S. Rahman and G. Vignale, Phys. Rev. B 30, 6951 (1984).

${ }^{17}$ W. Schülke and W. Lautner, Phys. Status Solidi B 66, 211 (1974).

${ }^{18}$ G. D. Priftis, J. Boviatsis, and A. Vradis, Phys. Lett. 68A, 482 (1978).

${ }^{19}$ A. Vradis and G. D. Priftis, Phys. Rev. B 32, 3556 (1985).

${ }^{20}$ W. Schülke, U. Bonse, H. Nagasawa, S. Mourikis, and A. Kaprolat, Phys. Rev. Lett. 59, 1361 (1987).

${ }^{21} \mathrm{G}$. Niklasson, Solid State Commun. 54, 665 (1985).

${ }^{22}$ T. K. Ng and B. Dabrowski, Phys. Rev. B 33, 5358 (1986).

${ }^{23}$ M. Taut and W. Hanke, Phys. Status Solidi B 77, 543 (1976).

${ }^{24}$ K. C. Pandey, P. M. Platzman, P. Eisenberger and E-Ni Foo, Phys. Rev. B 9, 5046 (1974).

${ }^{25}$ P. Nozieres and D. Pines, Phys. Rev. 113, 1254 (1959).

${ }^{26} \mathrm{D}$. Pines and P. Nozieres, The Theory of Quantum Liquids
(Benjamin, New York, 1966), Vol. 1.

${ }^{27}$ W. Schülke, U. Bonse, H. Nagasawa, A. Kaprolat, and A. Berthold, Phys. Rev. B 38, 2112 (1988).

${ }^{28}$ N. Wiser, Phys. Rev. 129, 62 (1963).

${ }^{29}$ E.-Ni Foo and J. J. Hopfield, Phys. Rev. 173, 635 (1968).

${ }^{30}$ K. Sturm and L. E. Oliviera, Phys. Rev. B 30, 4351 (1984).

${ }^{31}$ W. Schülke and H. Nagasawa, Nucl. Instrum. Methods 222, 203 (1984).

${ }^{32}$ W. Schülke, Nucl. Instrum. Methods A 246, 491 (1986).

${ }^{33}$ R. W. Shaw, J. Phys. C 2, 2350 (1969).

${ }^{34}$ J. Lindhard, K. Dan Vidensk. Selsk. Mat.-Fys. Medd. 28, 108 (1954).

${ }^{35}$ J. J. Quinn and R. A. Terrell, Phys. Rev. 112, 812 (1958).

${ }^{36}$ B. I. Lundquist, Phys. Kondens. Mater. 6, 206 (1967).

${ }^{37}$ J. Hubbard, Proc. R. Soc. London Ser. A 243, 336 (1957).

${ }^{38}$ S. Ichimaru and K. Utsumi, Phys. Rev. B 24, 7385 (1981).

${ }^{39}$ J. H. Tripp, P. M Everett, and W. L. Gordon, Phys. Rev. 180, 669 (1969).

${ }^{40}$ T. L. Loucks and P. H. Cutler, Phys. Rev. 133, A819 (1964).

${ }^{41}$ E. Clementi and C. Roetti, Atomic Data Nucl. Data Tables 14, 177 (1974).

${ }^{42}$ In-Whan Lyo and E. W. Plummer, Phys. Rev. Lett. 60, 1558 (1988).

${ }^{43}$ P. O. Nilsson, G. Arbman, and T. Gustafsson, J. Phys. F 4, 1937 (1974).

${ }^{44}$ M. Y. Chou, P. K. Lam, and M. L. Cohen, Phys. Rev. B 28, 4179 (1983). 